\title{
Buckling Analysis of Steel Fiber Column with Different Cross-Section and Boundary Conditions Using Euler-Bernoulli Beam Theory
}

\author{
M. Tunahan Özdemir ${ }^{a}$, Veysel Kobya ${ }^{b}$, Mustafa Ö̈gür Yayll ${ }^{c}$, Ali Mardani-Aghabaglou ${ }^{d}$ \\ ${ }^{a, b, c, d}$ Faculty of Engineering, Civil Engineering Departmant, Uludag University, Bursa, Turkey \\ E-mail adress: 501726009@ogr.uludag.edu.tr ${ }^{a}, \underline{511826008 @ \text { ogr.uludag.edu.tr }}{ }^{b}$, \\ ozguryayli@uludag.edu.tr ${ }^{c}$, alimardani@uludag.edu.tr ${ }^{d}$ \\ ORCID numbers of authors \\ 0000-0001-9313-9666 ${ }^{a}, 0000-0002-1226-8405^{b}, 0000-0003-2231-170 X^{c}, 0000-0003-0326-5015^{d}$
}

Received date: 22.02 .2019

Accepted date: 09.04.2019

\begin{abstract}
Nowadays, with the help of developing technology, engineering problems which are difficult to solve have become easily solved in a short time by means of computer software. Certain mathematical algorithms are used in these analysis methods. The mathematical and numerical solution methods created provide a significant solution facility for engineering. In this paper, the buckling analysis of the Euler column model, with elastic boundaries and containing steel fibers, under pressure effect is performed. In the column model, three different sections, which have been produced from four different concrete series, including three different types of fiber reinforced specimens and one non-fibrous control sample $(C)$ with $0.6 \%$ by volume, were analyzed by using a software. In the study, the analysis of the critical buckling values depends on length, elastic modulus and cross-sectional type of the column model has been performed. The results are shown in graphs and tables. With the results of the analysis, the effect of slenderness and steel fiber concrete on the critical load in pressure columns have been investigated.
\end{abstract}

Keywords: Elastically restrained ends, Euler columns, steel fiber reinforcement, comparison of buckling load

\section{Introduction}

Nowadays, with the developing technology, the results obtained from the engineering point of view are closer to reality. Analysis that are difficult to calculate are solved in a much shorter time with the help of computers and thus, it becomes easier to obtain different solution methods. In the light of these developments, various approaches have been presented to investigate the buckling behavior of structural elements under axial load. Due to various modelling, design and analysis difficulties with the finite element method, which is a method used for analyzing buckling behavior of structural elements in civil engineering, it is needed to use computer programs. At the beginning of the modelling and analysis process, Euler said that the pressure columns have been not only crushed but they also have stability problems [1]. Euler pioneered the researchers in analytical analysis of elastic columns and since the first study has been carried out in this area in 1744, the analysis has been called Euler load [2]. After Euler's first step for stability analysis, many researchers have contributed to the modelling of structural elements closer to reality. A.N. Dinnik [3] has designed the variable cross-section type of the column and 
beam elements, which is pinned from one end, clamped on the other end. And he examined the buckling behavior of them.

J.B. Keller [4] has studied the mechanical behavior of the circular cross-section and equilateral triangular section column and obtained results for the nonlinear buckling problem. Mechanical behaviors for similar Euler columns have been examined by I. Tadjbakhsh \& J.B. Keller, [5] and J.E. Taylor, [6], and exact results have been obtained for different boundary conditions. S. Timoshenko [7] has contributed to the realistic results by adding slip and rotational momentum factors in the beam to the Euler-Bernoulli theory Lee et al. [8-11] on the buckling loads, have made studies suitable for use in design. Q. Li, H. Cao, \& G. Li, [12], L. Qiusheng, C. Hong \& L. Guiqing [13] and Q. Li, H. Cao, \& G. Li, [14], in their work, using Bessel functions and super geometric functions, gave the flexural formulation of elastic columns with variable crosssection. He has stated that the axial load and bending stiffness distribution of the high structures are appropriately described. Kim and Kim [15] have presented a method of calculating the vibration frequency of beams in boundary conditions created by using the Fourier series, which is generally restrained and non-classical limiting methods of rotation and cycle springs.

M.T. Atay \& S.B. Coşkun [16], S.B. Coşkun \& M.T. Atay [17] have formed a column model with variable section and an elastically constrained along the upper and lower end and performed stability analysis with the Variational Iteration Method (VIM). K.V. Singh \& G. Li [18] in their study, they have created mathematical modelling of the Transcendental Eigenvalue Problem (TOE) using the previously proposed NEIM (numerical algorithm) numerical algorithm of axially graded and elastically constrained columns. He has performed a buckling analysis. The researchers has used many analytical and numerical methods to model column and beam elements and compared their results with experimental results or to each other [1933], similar to the approaches used in the column elements have been also studied in the beam elements [34-41].

Gül and Aydoğlu [42], in their study, beams on elastic foundation have examined in terms of the wave number vibrations with Euler-Bernoulli and Timoshenko beam theories using Hamilton's Principle. In the study by Y. Zhang et al. [43], using the kp-Ritz method for numerical solutions in buckling analysis, have been investigated the graphene layers in which the elastic medium has been modelled by the Winkler method. M.Ö. Yaylı [44] modelled the Euler column, which is bounded along its length and had different boundary conditions at its end points. In this numerical modelling, Stokes' transformations and Furier series have been used to create the coefficients matrix.

In this study, column model which is investigated by M.Ö. Yayl1 [44] has been created by using Euler's column theory. Firstly, the equation of the well-known Euler-Bernoulli beam theory in the literature is given under axial loads. Fourier sine series of displacement function is chosen to obtain analytical general solutions by M.Ö. Yayl1 [44]. Elasticity modules for EulerBernoulli column type structural elements with an external load applied at the upper and lower ends of the column with different boundary conditions have been calculated experimentally. In three different column sections produced from four different concrete series as, F30, F35, F50 fiber reinforced concrete and non-fibrous (C) sample, buckling analysis by using Mathematica program is made by taking the weight of the column element into account.

\section{Numerical Modelling of the Column to be Analyzed}

Fig. 1 shows the Euler column model which is elastically limited along the length of the column and has different boundary conditions. Government equation for buckling Timoshenko and Gere [45] and Wang et al. [46]: 


$$
\frac{d^{2}}{\mathrm{dx}^{2}}\left[\mathrm{EI} \frac{d^{2} y}{\mathrm{dx}^{2}}\right]+P \frac{d^{2} y}{\mathrm{dx}^{2}}+k_{w} y=0
$$

In the equation, EI is the bending stiffness, $\mathrm{P}$ is the axial load applied from the column end points, $k_{w}$ is the elastic limitation coefficient known as Winklers' constant applied for each unit length along the column length. M.Ö. Yaylı [44] in his study, obtained a matrix of coefficients representing the column model with a study of Stokes' transformations for the development of stability equations which limited the lateral displacements of the Furier sinus series. With this approach, it was stated that the buckling analysis of the Euler columns would yield closer results to the truth under different boundary conditions.



Fig. 1. An elastically restrained Euler column model.

Displacement function to apply Stokes' transformation:

$$
y(x)=\left\{\begin{array}{cc}
\delta_{0} & x=0 \\
\delta_{L} & x=L \\
\sum_{n=1}^{\infty} C_{n} \sin \left[\frac{n \pi x}{L}\right] & 0<x>L
\end{array}\right\}
$$

The Stokes transform are obtained using the displacement function and the Fourier series by M.Ö. Yayl1 [44];

$$
\mathrm{y}(x)=\sum_{n=1}^{\infty} \frac{2 \alpha_{n}\left((-1)^{\mathrm{n}}\left(\delta_{L}\left(\mathrm{P}-\mathrm{EI} \alpha_{n}{ }^{2}\right)+\mathrm{EI}_{L}{ }^{\prime \prime}\right)+\delta_{0}\left(\mathrm{EI} \alpha_{n}{ }^{2}-\mathrm{P}\right)-\mathrm{EI} \delta_{0}{ }^{\prime \prime}\right)}{\mathrm{L}\left(\mathrm{EI} \alpha_{n}{ }^{4}+k_{w}-\mathrm{P} \alpha_{n}{ }^{2}\right)} \sin \left(\frac{\mathrm{n} \pi x}{\mathrm{~L}}\right)
$$

Four homogeneous equations are obtained by applying the boundary [44]:

- Boundary conditions:

$$
\begin{gathered}
K_{0} \delta_{0}=-\mathrm{EI} \frac{\mathrm{d}^{3} y(x)}{\mathrm{d} x^{3}}, \quad \mathrm{x}=0 \\
K_{L} \delta_{L}=-\mathrm{EI} \frac{\mathrm{d}^{3} y(x)}{\mathrm{d} x^{3}}, \mathrm{x}=\mathrm{L} \\
\theta_{0} \frac{\mathrm{dy}(x)}{\mathrm{dx}}=\mathrm{EI} \frac{\mathrm{d}^{2} y(x)}{\mathrm{d} x^{2}}, \mathrm{x}=0 \\
\theta_{L} \frac{\mathrm{dy}(x)}{\mathrm{dx}}=-\mathrm{EI} \frac{\mathrm{d}^{2} y(x)}{\mathrm{d} x^{2}}, \mathrm{x}=\mathrm{L}
\end{gathered}
$$


- Homogeneous equations:

$$
\begin{aligned}
& \left(-\bar{K}_{0}-\sum_{n=1}^{\infty} \frac{2 n^{2} \pi^{2} \overline{K_{w}}}{n^{4} \pi^{4}-\bar{P}_{b} n^{2} \pi^{2}+K_{w}}\right) \frac{\delta_{0}}{L^{2}}+\left(\sum_{n=1}^{\infty} \frac{2 n^{2}(-1)^{n} \pi^{2} \overline{K_{w}}}{n^{4} \pi^{4}-\bar{P}_{b} n^{2} \pi^{2}+K_{w}}\right) \frac{\delta_{L}}{L^{2}}+ \\
& \left(1+\sum_{n=1}^{\infty} \frac{2\left(\bar{K}_{W}-\bar{P}_{b} n^{2} \pi^{2}\right)}{n^{4} \pi^{4}-\bar{P}_{b} n^{2} \pi^{2}+K_{w}}\right) \delta_{0}{ }^{\prime \prime}+\left(1+\sum_{n=1}^{\infty} \frac{2(-1)^{n}\left(\bar{K}_{W}-\bar{P}_{b} n^{2} \pi^{2}\right)}{n^{4} \pi^{4}-\bar{P}_{b} n^{2} \pi^{2}+K_{w}}\right) \delta_{L}{ }^{\prime \prime}=0 \\
& \left(\sum_{n=1}^{\infty} \frac{2 n^{2}(-1)^{n} \pi^{2} \overline{K_{w}}}{n^{4} \pi^{4}-\bar{P}_{b} n^{2} \pi^{2}+K_{w}}\right) \frac{\delta_{0}}{L^{2}}+\left(-\bar{K}_{L}-\sum_{n=1}^{\infty} \frac{2 n^{2} \pi^{2} \bar{K}_{w}}{n^{4} \pi^{4}-\bar{P}_{b} n^{2} \pi^{2}+K_{w}}\right) \frac{\delta_{L}}{L^{2}} \\
& +\left(1+\sum_{n=1}^{\infty} \frac{2(-1)^{n}\left(\overline{K_{w}}-\bar{P}_{b} n^{2} \pi^{2}\right)}{n^{4} \pi^{4}-\bar{P}_{b} n^{2} \pi^{2}+K_{w}}\right) \delta_{0}{ }^{\prime}+\left(1+\sum_{n=1}^{\infty} \frac{2\left(\overline{K_{w}}-\bar{P}_{b} n^{2} \pi^{2}\right)}{n^{4} \pi^{4}-\bar{P}_{b} n^{2} \pi^{2}+K_{w}}\right) \delta_{L}{ }^{\prime}=0 \\
& -\left(\overline{\theta_{0}}+2 \overline{\theta_{0}} \sum_{n=1}^{\infty} \frac{\overline{K_{w}}}{n^{4} \pi^{4}-\bar{P}_{b} n^{2} \pi^{2}+K_{w}}\right) \frac{\delta_{0}}{L^{2}}+\left(\overline{\theta_{0}}+2 \overline{\theta_{0}} \sum_{n=1}^{\infty} \frac{(-1)^{n} \overline{K_{w}}}{n^{4} \pi^{4}-\bar{P}_{b} n^{2} \pi^{2}+K_{w}}\right) \frac{\delta_{L}}{L^{2}} \\
& +\left(-1-2 \overline{\theta_{0}} \sum_{n=1}^{\infty} \frac{n^{2} \pi^{2}}{n^{4} \pi^{4}-\bar{P}_{b} n^{2} \pi^{2}+K_{w}}\right) \delta_{0}{ }^{\prime \prime}+\left(2 \overline{\theta_{0}} \sum_{n=1}^{\infty} \frac{n^{2} \pi^{2}(-1)^{n}}{n^{4} \pi^{4}-\bar{P}_{b} n^{2} \pi^{2}+K_{w}}\right) \delta_{L}{ }^{\prime \prime}=0 \\
& -\left(\overline{\theta_{L}}+2 \overline{\theta_{L}} \sum_{n=1}^{\infty} \frac{(-1)^{n} \overline{K_{W}}}{n^{4} \pi^{4}-\bar{P}_{b} n^{2} \pi^{2}+K_{w}}\right) \frac{\delta_{0}}{L^{2}}+\left(\overline{\theta_{L}}+2 \overline{\theta_{L}} \sum_{n=1}^{\infty} \frac{\overline{K_{W}}}{n^{4} \pi^{4}-\bar{P}_{b} n^{2} \pi^{2}+K_{w}}\right) \frac{\delta_{L}}{L^{2}}+ \\
& \left(2 \overline{\theta_{L}} \sum_{n=1}^{\infty} \frac{n^{2} \pi^{2}(-1)^{n}}{n^{4} \pi^{4}-\bar{P}_{b} n^{2} \pi^{2}+K_{w}}\right) \delta_{0}{ }^{\prime}+\left(-1-2 \overline{\theta_{L}} \sum_{n=1}^{\infty} \frac{n^{2} \pi^{2}}{n^{4} \pi^{4}-\bar{P}_{b} n^{2} \pi^{2}+K_{w}}\right) \delta_{L}{ }^{\prime}=0
\end{aligned}
$$

Where

$$
\bar{\theta}_{L}=\frac{\theta_{L} \mathrm{~L}}{\mathrm{EI}}, \bar{\theta}_{0}=\frac{\theta_{0} \mathrm{~L}}{\mathrm{EI}}, \overline{K_{0}}=\frac{K_{0} L^{3}}{\mathrm{EI}}, \bar{K}_{L}=\frac{K_{L} L^{3}}{\mathrm{EI}}, \overline{K_{w}}=\frac{k_{w} L^{4}}{\mathrm{EI}}, \quad \bar{P}_{b}=\frac{\mathrm{P} L^{2}}{\mathrm{EI}}
$$

Equations are written in matrix form M.Ö. Yayl1 [44]:

$$
\left[\begin{array}{llll}
\psi_{11} & \psi_{12} & \psi_{13} & \psi_{14} \\
\psi_{21} & \psi_{22} & \psi_{23} & \psi_{24} \\
\psi_{31} & \psi_{32} & \psi_{33} & \psi_{34} \\
\psi_{41} & \psi_{42} & \psi_{43} & \psi_{44}
\end{array}\right]\left[\begin{array}{c}
\frac{\delta_{0}}{L^{2}} \\
\frac{\delta_{L}}{L^{2}} \\
\delta_{0}^{\prime \prime} \\
\delta_{L}^{\prime \prime}
\end{array}\right]=0
$$

The eigenvalue system gives the critical buckling load M.Ö. Yaylı [44].

$$
\left|\psi_{i j}\right|=0, \quad(\mathrm{i}, \mathrm{j}=1,2,3,4)
$$

Where

$$
\begin{aligned}
& \psi_{11}=-\overline{K_{0}}-\sum_{n=1}^{\infty} \frac{2 n^{2} \pi^{2} \overline{K_{w}}}{n^{4} * \pi^{4}-\overline{P_{b}} n^{2} * \pi^{2}+\overline{K_{w}}}, \quad \quad \psi_{12}=\sum_{n=1}^{\infty} \frac{2 n^{2}(-1)^{n} \pi^{2} \overline{K_{w}}}{n^{4} * \pi^{4}-\bar{P}_{b} * n^{2} * \pi^{2}+\bar{K}_{w}} \\
& \psi_{12}=1+\sum_{n=1}^{\infty} \frac{2\left(\overline{K_{w}}-\overline{P_{b}} n^{2} \pi^{2}\right)}{n^{4} * \pi^{4}-\overline{P_{b} * n^{2} * \pi^{2}+\overline{K_{w}}}}, \quad \psi_{14}=1+\sum_{n=1}^{\infty} \frac{2(-1)^{n}\left(\overline{K_{w}}-\overline{P_{b}} n^{2} \pi^{2}\right)}{n^{4} * \pi^{4}-\overline{P_{b} * n^{2} * \pi^{2}+\overline{K_{w}}}}
\end{aligned}
$$




$$
\begin{aligned}
& \psi_{21}=\sum_{n=1}^{\infty} \frac{2 n^{2}(-1)^{n} \overline{K_{w}} \pi^{2}}{n^{4} * \pi^{4}-\overline{P_{b}} * n^{2} * \pi^{2}+\overline{K_{w}}}, \quad \psi_{22}=-\overline{K_{L}}-\sum_{n=1}^{\infty} \frac{2 n^{2} \overline{K_{w}} \pi^{2}}{n^{4} * \pi^{4}-\overline{P_{b}} * n^{2} * \pi^{2}+\bar{K}_{w}} \\
& \psi_{23}=1+\sum_{n=1}^{\infty} \frac{2(-1)^{n}\left(\overline{K_{w}}-\overline{P_{b}} n^{2} \pi^{2}\right)}{n^{4} * \pi^{4}-\overline{P_{b}} * n^{2} * \pi^{2}+\overline{K_{W}}}, \quad \psi_{24}=1+\sum_{n=1}^{\infty} \frac{2\left(\overline{K_{W}}-\overline{P_{b}} n^{2} \pi^{2}\right)}{n^{4} * \pi^{4}-\bar{P}_{b} * n^{2} * \pi^{2}+\bar{K}_{w}}
\end{aligned}
$$

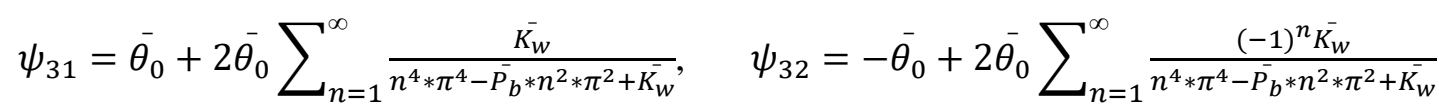

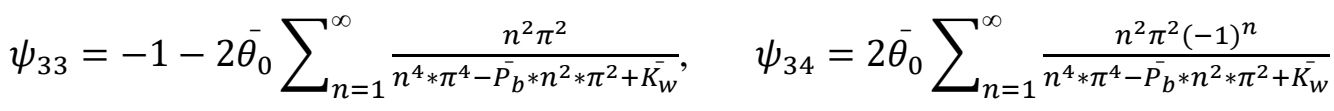



$$
\begin{aligned}
& \psi_{43}=2 \overline{\theta_{L}} \sum_{n=1}^{\infty} \frac{n^{2} \pi^{2}(-1)^{n}}{\overline{n^{4} * \pi^{4}-\overline{P_{b}} * n^{2} * \pi^{2}+\overline{K_{w}}}}, \quad \psi_{44}=-1-2 \overline{\theta_{L}} \sum_{n=1}^{\infty} \frac{n^{2} \pi^{2}}{\overline{n^{4} * \pi^{4}-\overline{P_{b}} * n^{2} * \pi^{2}+\bar{K}_{W}}}
\end{aligned}
$$

\section{Buckling Analysis of the Modelled Column}

In this section of the study, the figures given in Fig. 2. were selected for the sections of the column model to be analyzed. The cross-sectional features may vary according to the analysis.
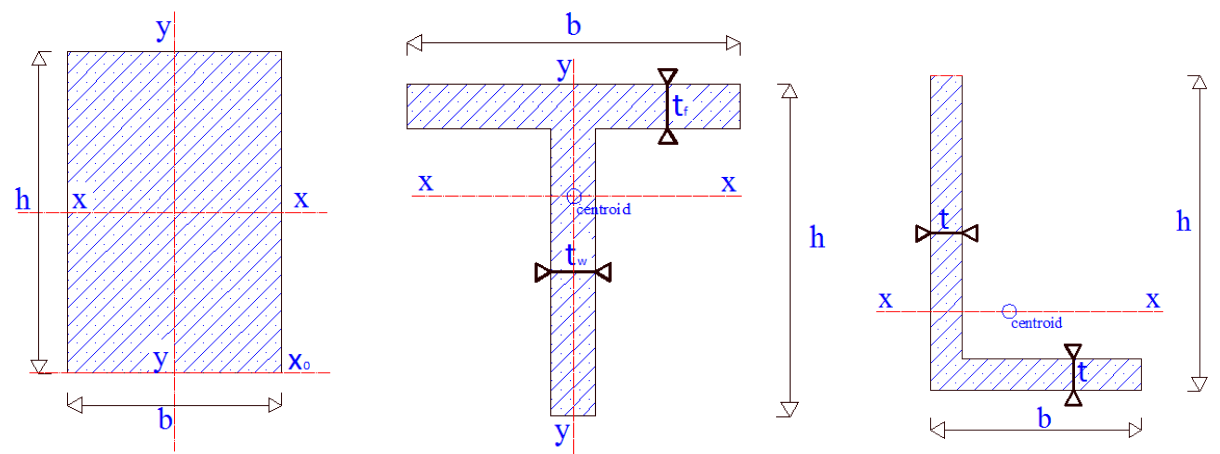

Fig. 2. Rectangular area, Tee section, Angle section, respectively.

The values of the elastic modulus calculated from the stress-strain graph obtained under 28 days pressure loading of each cylinder samples which has dimension of 10x20 cm are shown in Table 1.

Table 1. Elasticity modulus values depending on the type of steel fiber obtained as a result of the experiment.

\begin{tabular}{lllll}
\hline & C & F30 & F35 & F50 \\
Elasticity Module (Gpa) & 42.56 & 39.32 & 36.82 & 40.97 \\
\hline
\end{tabular}

\subsection{The effect of the rotation coefficient on the critical load}

The cross-sectional dimensions of the above column are used for the column to be analyzed. In the analysis, the modulus of elasticity was chosen as $\mathrm{E}=42560 \frac{\mathrm{N}}{\mathrm{mm}^{2}}$ column length $\mathrm{L}=3300$ mm. 200 terms were used in Stokes' transformations to provide the boundary condition of the 
Furier series. The number of terms is valid only for this analysis. The following cross-sectional measurements were used in the analysis: Rectangular area; $\mathrm{b}=600 \mathrm{~mm}, \mathrm{~h}=300 \mathrm{~mm}$; Tee section; $t_{f}=200 \mathrm{~mm}, t_{w}=200 \mathrm{~mm}, \mathrm{~h}=600 \mathrm{~mm}, \mathrm{~b}=600 \mathrm{~mm}$; Angle section $\mathrm{b}=600 \mathrm{~mm}, \mathrm{t}=200 \mathrm{~mm}, \mathrm{~h}=600$ $\mathrm{mm}$.

Table 2. Critical load values due to non-rotating spring coefficient $\left(K_{w}=0.0\right)$. (a)

\begin{tabular}{cccc}
\hline$\overline{\boldsymbol{\theta}_{\mathbf{0}}}=\overline{\boldsymbol{\theta}_{\mathbf{L}}}$ & $\boldsymbol{P}_{\boldsymbol{c r}} \mathbf{N}$ Rectangular section & $\boldsymbol{P}_{\boldsymbol{c r}} \mathbf{N}$ Tee section & $\boldsymbol{P}_{\boldsymbol{c r}} \mathbf{N}$ Angle section \\
\hline $\mathbf{0 . 0 0 1}$ & $5.20935 \times 10^{7}$ & $1.49206 \times 10^{8}$ & $5.96824 \times 10^{8}$ \\
$\mathbf{0 . 0 1}$ & $5.22832 \times 10^{7}$ & $1.49749 \times 10^{8}$ & $5.98998 \times 10^{8}$ \\
$\mathbf{0 . 1}$ & $5.41618 \times 10^{7}$ & $1.55130 \times 10^{8}$ & $6.20520 \times 10^{8}$ \\
$\mathbf{0 . 3}$ & $5.82171 \times 10^{7}$ & $1.66745 \times 10^{8}$ & $6.66981 \times 10^{8}$ \\
$\mathbf{0 . 5}$ & $6.21136 \times 10^{7}$ & $1.77905 \times 10^{8}$ & $7.11622 \times 10^{8}$ \\
$\mathbf{0 . 7 5}$ & $6.67711 \times 10^{7}$ & $1.91246 \times 10^{8}$ & $7.64982 \times 10^{8}$ \\
$\mathbf{1 . 0}$ & $7.12036 \times 10^{7}$ & $2.03941 \times 10^{8}$ & $8.15765 \times 10^{8}$ \\
$\mathbf{2 . 0}$ & $8.69193 \times 10^{7}$ & $2.48954 \times 10^{8}$ & $9.95816 \times 10^{8}$ \\
$\mathbf{4 . 0}$ & $1.10729 \times 10^{8}$ & $3.17149 \times 10^{8}$ & $1.26860 \times 10^{9}$ \\
$\mathbf{1 0 . 0}$ & $1.49022 \times 10^{8}$ & $4.26828 \times 10^{8}$ & $1.70731 \times 10^{9}$ \\
$\mathbf{2 0 . 0}$ & $1.73554 \times 10^{8}$ & $4.97092 \times 10^{8}$ & $1.98837 \times 10^{9}$ \\
$\mathbf{4 0 . 0}$ & $1.89774 \times 10^{8}$ & $5.43551 \times 10^{8}$ & $2.17421 \times 10^{9}$ \\
$\mathbf{8 0 . 0}$ & $1.99054 \times 10^{8}$ & $5.70130 \times 10^{8}$ & $2.28052 \times 10^{9}$ \\
$\mathbf{1 6 0 . 0}$ & $2.03995 \times 10^{8}$ & $5.84283 \times 10^{8}$ & $2.33713 \times 10^{9}$ \\
$\mathbf{3 0 0 . 0}$ & $2.06370 \times 10^{8}$ & $5.91084 \times 10^{8}$ & $2.36434 \times 10^{9}$ \\
\hline
\end{tabular}

Table 3. Critical load values due to non-rotating spring coefficient $\left(K_{w}=4.9\right)$. (b)

\begin{tabular}{cccc}
\hline$\overline{\boldsymbol{\theta}_{\mathbf{0}}}=\overline{\boldsymbol{\theta}_{\mathbf{L}}}$ & $\boldsymbol{P}_{\boldsymbol{c r}} \mathbf{N}$ Rectangular section & $\boldsymbol{P}_{\boldsymbol{c r}}$ N Tee section & $\boldsymbol{P}_{\boldsymbol{c r}}$ N Angle section \\
\hline $\mathbf{5}$ & $1.22341 \times 10^{8}$ & $3.50410 \times 10^{8}$ & $1.40164 \times 10^{9}$ \\
$\mathbf{1 0}$ & $1.51423 \times 10^{8}$ & $4.33706 \times 10^{8}$ & $1.73483 \times 10^{9}$ \\
$\mathbf{1 5}$ & $1.66635 \times 10^{8}$ & $4.77276 \times 10^{8}$ & $1.90911 \times 10^{9}$ \\
$\mathbf{2 0}$ & $1.75805 \times 10^{8}$ & $5.03540 \times 10^{8}$ & $2.01416 \times 10^{9}$ \\
$\mathbf{2 5}$ & $1.81888 \times 10^{8}$ & $5.20964 \times 10^{8}$ & $2.08386 \times 10^{9}$ \\
$\mathbf{3 0}$ & $1.86203 \times 10^{8}$ & $5.33323 \times 10^{8}$ & $2.13329 \times 10^{9}$ \\
$\mathbf{3 5}$ & $1.89418 \times 10^{8}$ & $5.42529 \times 10^{8}$ & $2.17012 \times 10^{9}$ \\
$\mathbf{4 0}$ & $1.91902 \times 10^{8}$ & $5.49644 \times 10^{8}$ & $2.19858 \times 10^{9}$ \\
$\mathbf{4 5}$ & $1.93878 \times 10^{8}$ & $5.55305 \times 10^{8}$ & $2.22122 \times 10^{9}$ \\
$\mathbf{5 0}$ & $1.95487 \times 10^{8}$ & $5.59913 \times 10^{8}$ & $2.23965 \times 10^{9}$ \\
$\mathbf{5 5}$ & $1.96822 \times 10^{8}$ & $5.63737 \times 10^{8}$ & $2.25495 \times 10^{9}$ \\
$\mathbf{6 0}$ & $1.97947 \times 10^{8}$ & $5.66960 \times 10^{8}$ & $2.26784 \times 10^{9}$ \\
$\mathbf{6 5}$ & $1.98908 \times 10^{8}$ & $5.69713 \times 10^{8}$ & $2.27885 \times 10^{9}$ \\
$\mathbf{7 0}$ & $1.99739 \times 10^{8}$ & $5.72092 \times 10^{8}$ & $2.28837 \times 10^{9}$ \\
$\mathbf{7 5}$ & $2.00464 \times 10^{8}$ & $5.74168 \times 10^{8}$ & $2.29667 \times 10^{9}$ \\
\hline
\end{tabular}




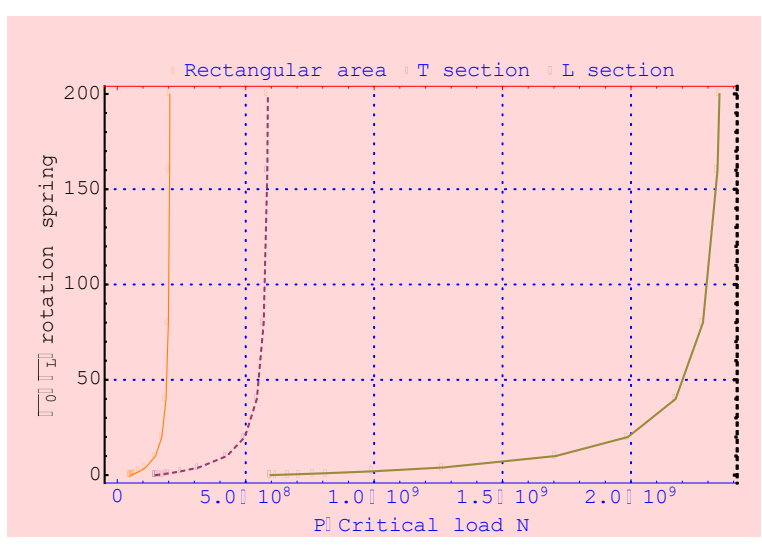

a.

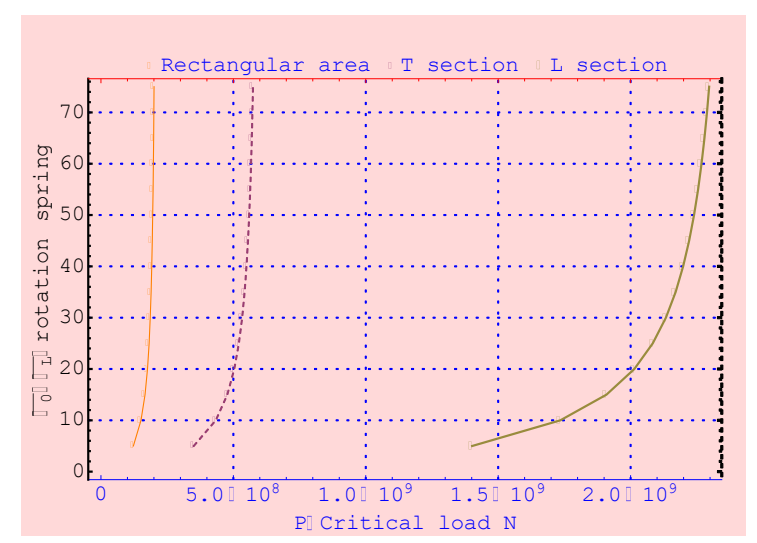

b.

Fig. 3. Graph of change in critical load values due to non-rotating spring coefficient.

When graphs and tables are examined, it is seen that the values of the spring $\left(\overline{\theta_{0}}\right.$ and $\left.\overline{\theta_{\mathrm{L}}}\right)$ constant which prevent the rotation of the column ends owing to increase the stiffness of the column ends. This increase is a hyperbolically increasing curve from a clamped support to pinned support behavior. Because of the increase in the values taken by the spring constant after a certain point of rigidity, we can call this column clamped-clamped columns. It can be said that the critical load values are examined according to the spring constant that prevents rotation, i.e. according to the boundary condition of the column. As the spring $\left(\overline{\theta_{0}}\right.$ and $\left.\overline{\theta_{\mathrm{L}}}\right)$ constant value increased the critical load value increased in the column, likewise as it decreases the critical load decreased. This non-linearly increased critical load reached a maximum critical load point after a certain $\overline{\theta_{0}}$ and $\overline{\theta_{\mathrm{L}}}$ value. At this point, it can be said that both ends of the column are clamped because, as theoretically, the ends are not allowed to rotate.

\subsection{Critical load change depending on $\mathrm{L} / \mathrm{d}$ ratio}

For the column model, which is calculated according to the $\mathrm{L} / \mathrm{d}$ ratio in table 1 , the values of $\mathrm{A}=\mathrm{b} . \mathrm{h}$, inertial radius $\mathrm{d}=\sqrt{I / A}, \overline{K_{\mathrm{L}}}=0.0, \mathrm{~b}=600 \mathrm{~mm}, \mathrm{~h}=400 \mathrm{~mm}$ were selected for the column model. Modulus of elasticity obtained from the experiment results is $E=42560 \frac{\mathrm{N}}{\mathrm{mm}^{2}}$. 150 terms have been used in Stokes transformations to provide the boundary condition of Furier series. The number of terms is valid only for this analysis. The spring constant values $\left(\overline{\theta_{0}}=0.00000001, \overline{\theta_{\mathrm{L}}}=100000000\right)$ at the column ends are selected as very high or near zero values for the boundary conditions. Thus, the fixed and clamped support model which is required for the column is obtained.
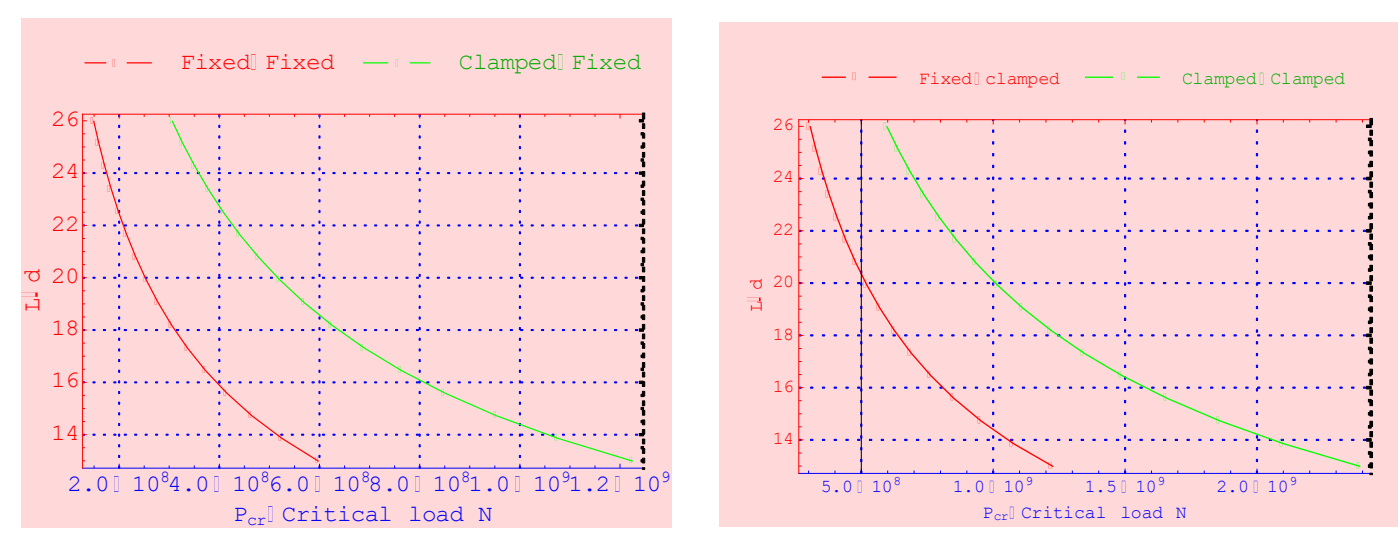

Fig. 4. Critical load graph due to L/d ratio in different boundary conditions. 
Table 4. The effect of column thickness on critical load.

\begin{tabular}{cccc}
\hline \multicolumn{1}{c}{ /d } & $\boldsymbol{P}_{\boldsymbol{c r}}$ Fixed-Fixed N & $\boldsymbol{P}_{\boldsymbol{c r}}$ Fixed-Clamped N & $\boldsymbol{P}_{\boldsymbol{c r}}$ Clamped-Clamped N \\
\hline $\mathbf{2 5 . 9 8 0 7 6 2 1}$ & $1.49351 \times 10^{8}$ & $3.06361 \times 10^{8}$ & $5.97405 \times 10^{8}$ \\
$\mathbf{2 5 . 1 1 4 7 3 6 7}$ & $1.59829 \times 10^{8}$ & $3.27853 \times 10^{8}$ & $6.39316 \times 10^{8}$ \\
$\mathbf{2 4 . 2 4 8 7 1 1 3}$ & $1.71449 \times 10^{8}$ & $3.51690 \times 10^{8}$ & $6.85797 \times 10^{8}$ \\
$\mathbf{2 3 . 3 8 2 6 8 5 9}$ & $1.84384 \times 10^{8}$ & $3.78223 \times 10^{8}$ & $7.37537 \times 10^{8}$ \\
$\mathbf{2 2 . 5 1 6 6 6 0 5}$ & $1.98840 \times 10^{8}$ & $4.07877 \times 10^{8}$ & $7.95362 \times 10^{8}$ \\
$\mathbf{2 1 . 6 5 0 6 3 5 1}$ & $2.15066 \times 10^{8}$ & $4.41159 \times 10^{8}$ & $8.60263 \times 10^{8}$ \\
$\mathbf{2 0 . 7 8 4 6 0 9 7}$ & $2.33361 \times 10^{8}$ & $4.78689 \times 10^{8}$ & $9.33445 \times 10^{8}$ \\
$\mathbf{1 9 . 9 1 8 5 8 4 3}$ & $2.54095 \times 10^{8}$ & $5.21219 \times 10^{8}$ & $1.01638 \times 10^{9}$ \\
$\mathbf{1 9 . 0 5 2 5 5 8 9}$ & $2.77719 \times 10^{8}$ & $5.69679 \times 10^{8}$ & $1.11088 \times 10^{9}$ \\
$\mathbf{1 8 . 1 8 6 5 3 3 5}$ & $3.04798 \times 10^{8}$ & $6.25226 \times 10^{8}$ & $1.21919 \times 10^{9}$ \\
$\mathbf{1 7 . 3 2 0 5 0 8 1}$ & $3.36040 \times 10^{8}$ & $6.89312 \times 10^{8}$ & $1.34416 \times 10^{9}$ \\
$\mathbf{1 6 . 4 5 4 4 8 2 7}$ & $3.72344 \times 10^{8}$ & $7.63780 \times 10^{8}$ & $1.48938 \times 10^{9}$ \\
$\mathbf{1 5 . 5 8 8 4 5 7 3}$ & $4.14865 \times 10^{8}$ & $8.51002 \times 10^{8}$ & $1.65946 \times 10^{9}$ \\
$\mathbf{1 4 . 7 2 2 4 3 1 9}$ & $4.65108 \times 10^{8}$ & $9.54064 \times 10^{8}$ & $1.86043 \times 10^{9}$ \\
$\mathbf{1 3 . 8 5 6 4 0 6 5}$ & $5.25063 \times 10^{8}$ & $1.07705 \times 10^{9}$ & $2.10025 \times 10^{9}$ \\
$\mathbf{1 2 . 9 9 0 3 8 1 1}$ & $5.97405 \times 10^{8}$ & $1.22544 \times 10^{9}$ & $2.38962 \times 10^{9}$ \\
\hline
\end{tabular}

When Table 1 and Fig. 4. are examined, it is seen that increase of L/d ratio causes decrease of critical load value and increase of $\mathrm{L} / \mathrm{d}$ ratio causes parabolic increase of critical load. ease of critical load value and increase of $\mathrm{L} / \mathrm{d}$ ratio causes parabolic increase of critical load. The increase in the $\mathrm{L} / \mathrm{d}$ ratio is result from an increase in column length or a smaller diameter in the cross-sectional area. Increased column diameter or decreased in height reduces the slenderness and therefore it can be said that there will be collapse caused by crushing instead of buckling in the column, because the column will be crushed before reaching the value of excessive critical buckling load. There are limitations in terms of slenderness in the relevant regulations. As can be seen from the limitation of the regulations, with the increase in the column length or the reduction of the column cross-section, the critical load value decreases and this result gives us information about the fact that there is a limit to making high columns with small sections relation with slenderness.

\subsection{Critical load change depending on the modulus of elasticity}

Table 5 shows the results of the analysis made according to the modulus of elasticity values obtained from the samples in the mixture ratios of different steel fiber previously made for the column model. 3 different cross-sectional types were used in the analysis. These sections are from Fig. 2.;

Rectangular $: \mathrm{b}=900 \mathrm{~mm}, \mathrm{~h}=600 \mathrm{~mm}$;

T section : $t_{f}=200 \mathrm{~mm}, t_{w}=200 \mathrm{~mm}, \mathrm{~h}=600 \mathrm{~mm}, \mathrm{~b}=600 \mathrm{~mm}$;

L section (Angle) : $b=600 \mathrm{~mm}, \mathrm{t}=200 \mathrm{~mm}, \mathrm{~h}=600 \mathrm{~mm}$;

spring coefficients:

$\overline{K_{0}}=100000000, \overline{K_{\mathrm{L}}}=100000000, \overline{\theta_{0}}=1000000000, \overline{\theta_{\mathrm{L}}}=1000000000, \overline{K_{\mathrm{w}}}=50.0$ 
Table 5. Critical load values connected to the E value of different sections with clamped-clamped ends.

\begin{tabular}{|c|c|c|}
\hline I $\mathrm{mm}^{4}$ & $\mathrm{E} \frac{N}{m m^{2}}$ & $P_{c r} \mathbf{N}$ \\
\hline $1,62 \times 10^{10}$ (Rectangular) & $\begin{array}{l}42560 \\
40970 \\
39320 \\
36820\end{array}$ & $\begin{array}{l}1.53916 \times 10^{9} \\
1.42202 \times 10^{9} \\
1.33163 \times 10^{9} \\
1.48167 \times 10^{9}\end{array}$ \\
\hline $3.86667 \times 10^{9}(\mathrm{~T}$ section $)$ & $\begin{array}{l}42560 \\
40970 \\
39320 \\
36820\end{array}$ & $\begin{array}{l}3.67456 \times 10^{8} \\
3.39485 \times 10^{8} \\
3.17901 \times 10^{8} \\
3.53730 \times 10^{8}\end{array}$ \\
\hline $1.54667 \times 10^{10}(\mathrm{~L}$ section $)$ & $\begin{array}{l}42560 \\
40970 \\
39320 \\
36820\end{array}$ & $\begin{array}{l}1.46951 \times 10^{9} \\
1.35767 \times 10^{9} \\
1.27137 \times 10^{9} \\
1.41462 \times 10^{9}\end{array}$ \\
\hline
\end{tabular}

spring coefficients:

$\overline{K_{0}}=100000000, \overline{K_{\mathrm{L}}}=100000000, \overline{\theta_{0}}=1000000000, \overline{\theta_{\mathrm{L}}} 1000000000, \overline{K_{\mathrm{w}}}=0.0 ;$

Table 6. Critical load values connected to the $\mathrm{E}$ value of different sections with pinned-pinned ends.

\begin{tabular}{|c|c|c|}
\hline I $\mathrm{mm}^{4}$ & $\mathrm{E} \frac{N}{m m^{2}}$ & $P_{c r} \mathbf{N}$ \\
\hline $1,62 \times 10^{10}$ (Rectangular) & $\begin{array}{l}42560 \\
40970 \\
39320 \\
36820\end{array}$ & $\begin{array}{l}2.74811 \times 10^{9} \\
2.64545 \times 10^{9} \\
2.53891 \times 10^{9} \\
2.37748 \times 10^{9}\end{array}$ \\
\hline $3.86667 \times 10^{9}(\mathrm{~T}$ section $)$ & $\begin{array}{l}42560 \\
40970 \\
39320 \\
36820\end{array}$ & $\begin{array}{l}6.55928 \times 10^{8} \\
6.31424 \times 10^{8} \\
6.05994 \times 10^{8} \\
5.67464 \times 10^{8}\end{array}$ \\
\hline $1.54667 \times 10^{10}(\mathrm{~L}$ section $)$ & $\begin{array}{l}42560 \\
40970 \\
39320 \\
36820\end{array}$ & $\begin{array}{l}2.62371 \times 10^{9} \\
2.52569 \times 10^{9} \\
2.42398 \times 10^{9} \\
2.26986 \times 10^{9}\end{array}$ \\
\hline
\end{tabular}

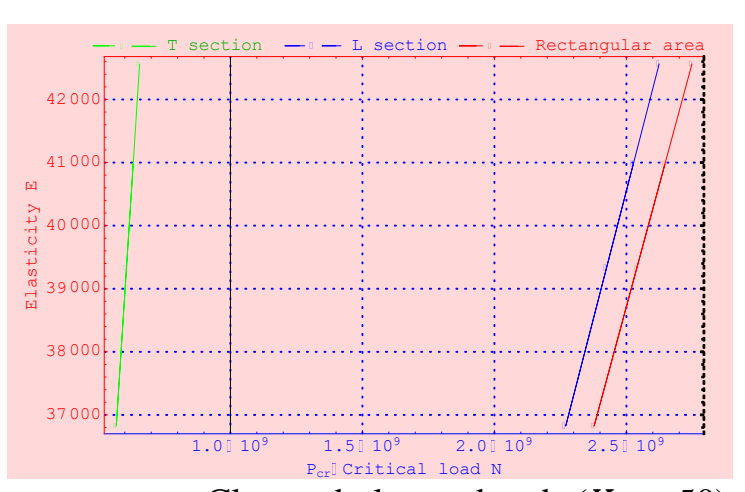

Clamped-clamped ends $\left(K_{w}=50\right)$.

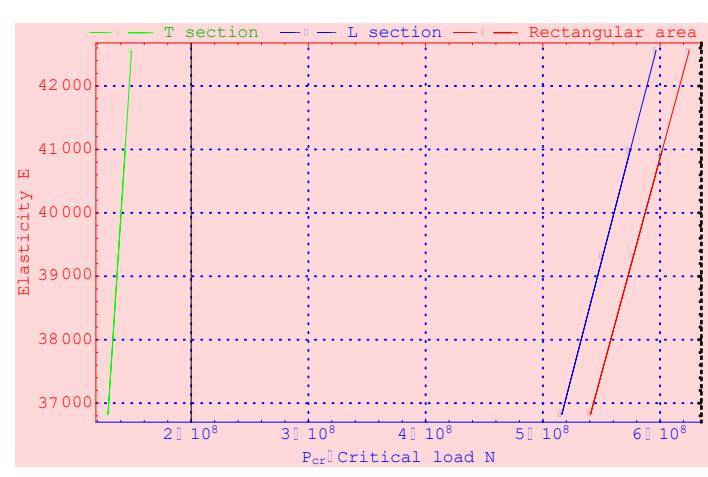

Pinned-pinned ends $\left(K_{w}=0\right)$.

Fig. 5. Critical load graphs connected to the Elasticity module in different section types of columns. 
When Fig. 5. Table 5. and Table 6. are examined, it is seen that the increase of the modulus of elasticity increases the critical load value linearly.

\subsection{Critical load value depending on the column length}

Table 5 shows the critical load values of 3 different section types in column-length analysis. Spring coefficients for the column model:

$\overline{\theta_{0}}=0.000000001, \overline{\theta_{\mathrm{L}}}=0.0000000001, \overline{K_{0}}=10000000, \overline{K_{\mathrm{L}}}=10000000 \overline{K_{\mathrm{w}}}=50.0$.

Modulus of elasticity obtained from the experiment results is $\mathrm{E}=42000 \frac{\mathrm{N}}{\mathrm{mm}^{2}} \cdot 150$ terms were used in the analysis.

Measurements of the sections to be used in the analysis:

Rectangular : $\mathrm{b}=600 \mathrm{~mm}, \mathrm{~h}=400 \mathrm{~mm}$;

Tee section : $t_{f}=200 \mathrm{~mm}, t_{w}=200 \mathrm{~mm}, \mathrm{~h}=600 \mathrm{~mm}, \mathrm{~b}=600 \mathrm{~mm}$;

L section (Angle) : $b=400 \mathrm{~mm}, \mathrm{t}=200 \mathrm{~mm}, \mathrm{~h}=400 \mathrm{~mm}$

Table 7. Table of critical load change due to column length of different sections with clamped-clamped

\begin{tabular}{cccc}
\multicolumn{4}{c}{ ends. } \\
\hline Column Length mm & $\begin{array}{c}\boldsymbol{P}_{\boldsymbol{c r}} \mathbf{N} \\
\text { Rectangular Area }\end{array}$ & $\begin{array}{c}\boldsymbol{P}_{\boldsymbol{c r}} \mathbf{N} \\
\text { T section }\end{array}$ & $\begin{array}{c}\boldsymbol{P}_{\boldsymbol{c r}} \mathbf{N} \\
\text { Angle section }\end{array}$ \\
\hline $\mathbf{3 0 0 0}$ & $6.56833 \times 10^{8}$ & $7.93673 \times 10^{8}$ & $9.85249 \times 10^{8}$ \\
$\mathbf{3 3 0 0}$ & $5.42837 \times 10^{8}$ & $6.55928 \times 10^{8}$ & $8.14256 \times 10^{8}$ \\
$\mathbf{3 6 0 0}$ & $4.56134 \times 10^{8}$ & $5.51162 \times 10^{8}$ & $6.84201 \times 10^{8}$ \\
$\mathbf{3 9 0 0}$ & $3.88659 \times 10^{8}$ & $4.69629 \times 10^{8}$ & $5.82988 \times 10^{8}$ \\
$\mathbf{4 2 0 0}$ & $3.35119 \times 10^{8}$ & $4.04935 \times 10^{8}$ & $5.02678 \times 10^{8}$ \\
$\mathbf{4 5 0 0}$ & $2.91926 \times 10^{8}$ & $3.52744 \times 10^{8}$ & $4.37889 \times 10^{8}$ \\
$\mathbf{4 8 0 0}$ & $2.56575 \times 10^{8}$ & $3.10029 \times 10^{8}$ & $3.84863 \times 10^{8}$ \\
$\mathbf{5 1 0 0}$ & $2.27278 \times 10^{8}$ & $2.74627 \times 10^{8}$ & $3.40917 \times 10^{8}$ \\
$\mathbf{5 4 0 0}$ & $2.02726 \times 10^{8}$ & $2.44961 \times 10^{8}$ & $3.04089 \times 10^{8}$ \\
$\mathbf{5 7 0 0}$ & $1.81948 \times 10^{8}$ & $2.19854 \times 10^{8}$ & $2.72922 \times 10^{8}$ \\
$\mathbf{6 0 0 0}$ & $1.64208 \times 10^{8}$ & $1.98418 \times 10^{8}$ & $2.46312 \times 10^{8}$ \\
\hline
\end{tabular}

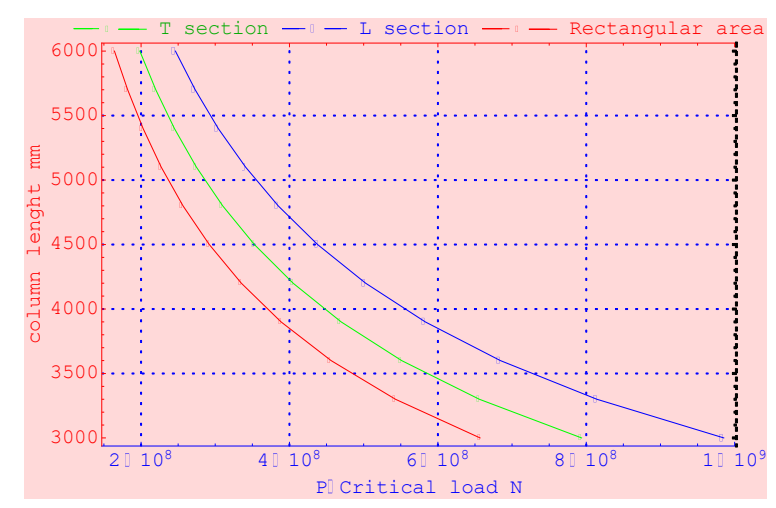

Fig. 6. Critical load variation due to column length of different sections with clamped-clamped ends.

Table 7. and Fig 6. shows that the effect of column length on critical load is not linear. Increasing the column length causes a hyperbolic reduction of the load capacity as it makes the column delicate. 


\subsection{Critical load disribution due to modulus of elasticity}

Three different cross-sectional types were used in the analyzes. $\overline{K_{w}}=0.0$ is selected spring coefficient for elastic restrained and $L=3300$ is selected for column length. Table 8 shows critical buckling values depending on the values given. Similarly, Table 9. shows the results of the critical buckling load to be obtained when the $\mathrm{L}=3300, \overline{K_{w}}=50.0, \overline{P_{b}}=\left(P_{c r} L^{2}\right) /(\mathrm{E} \mathrm{I})$, $\overline{K_{0}}=10000000, \overline{K_{L}}=10000000, \overline{\theta_{0}}=10000000, \overline{\theta_{L}}=100000000$ values are used for the column model to be analyzed. In the same way, table 8 . shows the moment of inertia that is based on section dimensions, elasticity modules and section dimensions. The graph of the critical buckling values found in figure 7 is shown.

Table 8. Critical load distribution table of the different sections connected to the modulus of elasticity with fixed-fixed ends.

\begin{tabular}{|c|c|c|c|c|c|}
\hline I $\mathrm{mm}^{4}$ & $\begin{array}{c}\text { sectional } \\
\text { dimensions } \\
\text { (bxh) mm }\end{array}$ & $\begin{array}{c}P_{c r} \mathrm{~N} \\
(\mathrm{E}=\mathbf{3 6 8 2 0} \\
\left.\mathrm{N} / \mathrm{mm}^{2}\right)\end{array}$ & $\begin{array}{c}P_{c r} \mathrm{~N} \\
(\mathrm{E}=39320 \\
\left.\mathrm{N} / \mathrm{mm}^{2}\right)\end{array}$ & $\begin{array}{c}P_{c r} \mathrm{~N} \\
(\mathrm{E}=40970 \\
\left.\mathrm{N} / \mathrm{mm}^{2}\right)\end{array}$ & $\begin{array}{c}P_{c r} \mathrm{~N} \\
(\mathrm{E}=42560 \\
\left.\mathrm{N} / \mathrm{mm}^{2}\right)\end{array}$ \\
\hline $1,350 \times 10^{9}$ & $(600 * 300)$ & $4.50494 \times 10^{7}$ & $4.81082 \times 10^{7}$ & $5.0127 \times 10^{7}$ & $5.20724 \times 10^{7}$ \\
\hline $3,200 \times 10^{9}$ & $(600 * 400)$ & $1.06784 \times 10^{8}$ & $1.14034 \times 10^{8}$ & $1.1882 \times 10^{8}$ & $1.23431 \times 10^{8}$ \\
\hline $6,250 \times 10^{9}$ & $(600 * 500)$ & $2.08562 \times 10^{8}$ & $2.22723 \times 10^{8}$ & $2.32069 \times 10^{8}$ & $2.41076 \times 10^{8}$ \\
\hline $1,080 \times 10^{10}$ & $(600 * 600)$ & $3.60396 \times 10^{8}$ & $3.84866 \times 10^{8}$ & $4.01016 \times 10^{8}$ & $4.16579 \times 10^{8}$ \\
\hline $1,715 \times 10^{10}$ & $(600 * 700)$ & $5.72295 \times 10^{8}$ & $6.11152 \times 10^{8}$ & $6.36798 \times 10^{8}$ & $6.61512 \times 10^{8}$ \\
\hline $2,560 \times 10^{10}$ & $(600 * 800)$ & $8.54271 \times 10^{8}$ & $9.12274 \times 10^{8}$ & $9.50556 \times 10^{8}$ & $9.87446 \times 10^{8}$ \\
\hline
\end{tabular}

Table 9. Critical load distribution table of the different sections connected to the modulus of elasticity with clamped-clamped ends.

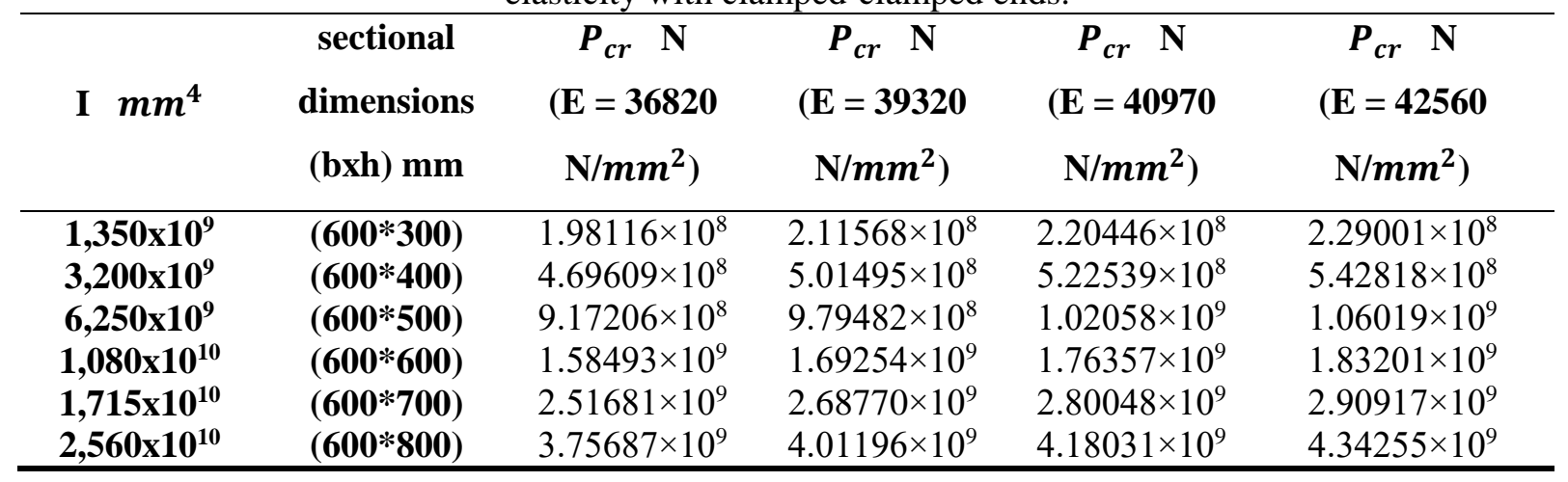

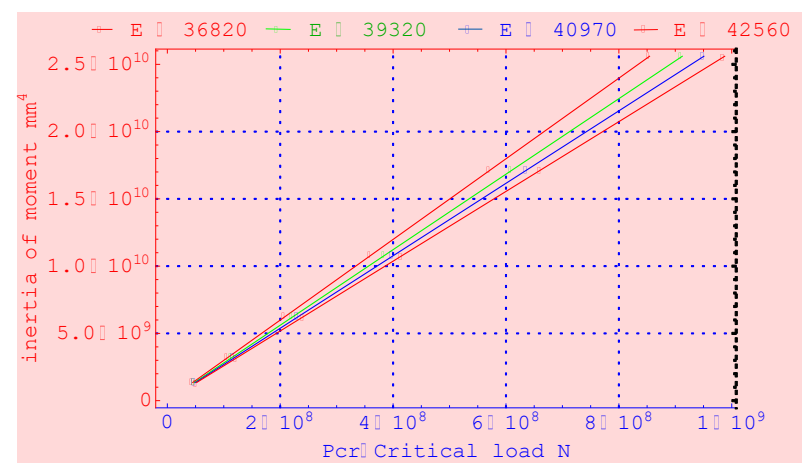

(a) Fixed-fixed

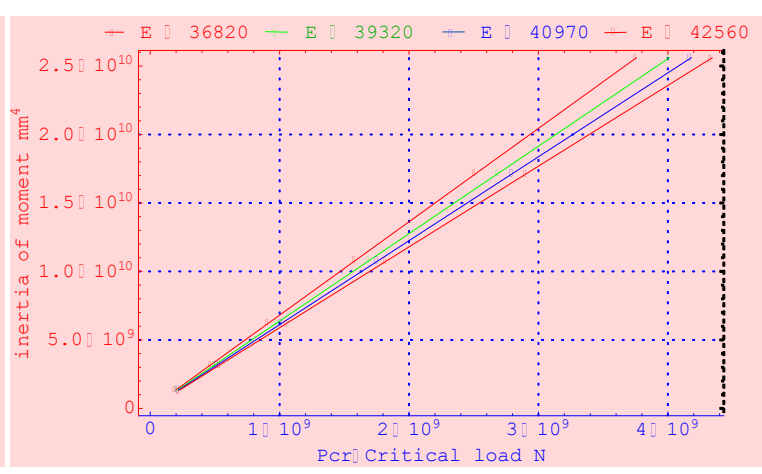

(b) Clamped-clamped

Fig. 7. Critical load distribution table of the different sections connected to the modulus of elasticity. 
Table 9. and Fig 7. shows that the increase in the section width leads to an increase in the critical load value. Similarly, the increase in the modulus of elasticity of the material also increased the value of the critical load.

\section{Conclusion}

In the study, the buckling analysis of the Euler column model with different boundary conditions, which was elastically limited a column model, with the help of Stokes' transformations and Fourier sine series which had been previously proposed by the researchers, was done. In the analysis, elasticity modulus values of the steel fiber reinforced and non-fibrous concrete specimens with different mixing ratios obtained from the experimental results were used. The status of three different sections was examined. The result show that:

- Because of increased column diameter or decrease in the length of the column, the critical buckling load values in the column increased hyperbolically. For this reason, very large critical buckling load values are obtained. This indicates that the crush will occur due to pressure load before a buckling occurs after a certain critical load value in the column. Regarding this, restrictions have been made in terms of delicacy in the regulations. In other words, with the increase in column length or narrowing of the diameter, the critical load value decreases and this result gives us information about the fact that there is a limit to making high columns with small sections.

- Since the value of the modulus of elasticity obtained from the non-fibrous concrete (C) mixture is the highest value, the highest value of the critical load is seen in this sample. Then the greatest critical load values were seen in F50, F30, and F35, respectively.

\section{Acknowledgment}

The authors gratefully acknowledge support provided by Bursa Uludag University Scientific Research Projects Centre (BAP) under grant numbers KUAP(MH)-2017/11.

The authors would like to thank Bursa-Beton Ready Mixed Concrete Plant and Polisan Construction Chemicals Company authorities for their kind assistance in providing the cement, superplasticizer admixture and fiber as well as determining the chemical composition of these products.

\section{References}

[1] Euler, L., De curvis elasticis. In Methodus inveniendi leneas curva maximi minimive proprietate gaudentes, sive solutio problematis isoperimetrici lattisimo sensu accepti, Lausannae, E65A. O. O. Ser.I., 24, 231-297, 1744.

[2] Brush, D. O., Almroth, B. O., Buckling of Bars, Plates and Shells, McGraw-Hill Comp., 1975.

[3] Dinnik, A. N., Design of columns of varying cross section. Trans ASME, 51(1), 105-14, 1929.

[4] Keller, J. B., The shape of the strongest column. Archive for Rational Mechanics and Analysis, 5(1), 275-285, 1960.

[5] Tadjbakhsh, I., \& Keller, J. B. Strongest columns and isoperimetric inequalities for eigenvalues. Journal of Applied Mechanics, 29(1), 159-164, 1962. 
[6] Taylor, J. E., The strongest column: an energy approach. Journal of Applied Mechanics, 34(2), 486-487, 1967.

[7] Timoshenko, S., Theory of elastic stability 2e. Tata McGraw-Hill Education, 1970.

[8] Lee, G.C., Morrell, M.L. and Ketter, R.L., "Design of Tapered Members" Welding Research Council, Bulletin \#173, June 1972.

[9] Lee, G.C., Chen, Y.C. and Hsu, T.L., "Allowable Axial Stress of Restrained MultiSegment, Tapered Roof Girders.” Welding Research Council, Bulletin \#248, May 1979.

[10] Lee, G.C. and Hsu, T.L., “Tapered Columns with Unequal Flanges.” Welding Research Council, Bulletin \#272, November 1981.

[11] Lee, G.C., Ketter, R.L. and Hsu, T.L., "Design of Single Story Rigid Frames", Metal Building Manufacturer's Association, Cleveland, Ohio, 1981.

[12] Li, Q., Cao, H., \& Li, G., Stability analysis of a bar with multi-segments of varying crosssection. Computers \& structures, 53(5), 1085-1089, 1994.

[13] Qiusheng, L., Hong, C., \& Guiqing, L., Stability analysis of bars with varying crosssection. International Journal of Solids and Structures, 32(21), 3217-3228, 1995.

[14] Li, Q., Cao, H., \& Li, G., Static and dynamic analysis of straight bars with variable crosssection. Computers \& structures, 59(6), 1185-1191, 1996.

[15] Kim, H. K., Kim, M. S., Vibration of beams with generally restrained boundary conditions using Fourier series. Journal of Sound Vibration 245(5):771-784. doi: 10.1006/jsvi.2001.3615, 2001.

[16] Atay, M. T., \& Coşkun, S., Elastic stability of Euler columns with a continuous elastic restraint using variational iteration method. Computers \& Mathematics with Applications, 58(11-12), 2528-2534, 2009.

[17] Coşkun, S. B., \& Atay, M. T., Determination of critical buckling load for elastic columns of constant and variable cross-sections using variational iteration method. Computers \& Mathematics with Applications, 58(11-12), 2260-2266, 2009.

[18] Singh, K. V., \& Li, G., Buckling of functionally graded and elastically restrained nonuniform columns. Composites Part B: Engineering, 40(5), 393-403, 2009.

[19] Yao, F., Meng, W., Zhao, J., She, Z., \& Shi, G., Analytical method comparison on critical force of the stepped column model of telescopic crane. Advances in Mechanical Engineering, 10(10), 1687814018808697, 2018.

[20] Atay, M. T. Determination of buckling loads of tilted buckled column with varying flexural rigidity using variational iteration method. International Journal of Nonlinear Sciences and Numerical Simulation, 11(2), 97-104, 2010. 
[21] Okay, F., Atay, M. T., \& Coşkun, S. B. Determination of buckling loads and mode shapes of a heavy vertical column under its own weight using the variational iteration method. International Journal of Nonlinear Sciences and Numerical Simulation, 11(10), 851-858, 2010.

[22] Al-Kamal, M. K., Estimating Elastic Buckling Load for an Axially Loaded Column Bolted to a Simply Supported Plate using Energy Method. Alnahrain journal for engineering sciences, 20(5), 1154-1159, 2017.

[23] Yilmaz, Y., Girgin, Z., \& Evran, S., Buckling analyses of axially functionally graded nonuniform columns with elastic restraint using a localized differential quadrature method. Mathematical Problems in Engineering, 2013.

[24] Ofondu, I. O., Ikwueze, E. U., \& Ike, C. C., Determination of the critical buckling loads of euler columns using stodola-vianello iteration method. Malaysian Journal of Civil Engineering, 30(3), 2018.

[25] Coşkun, S. B., Determination of critical buckling loads for euler columns of variable flexural stiffness with a continuous elastic restraint using homotopy perturbation method. International Journal of Nonlinear Sciences and Numerical Simulation, 10(2), 191-198, 2009.

[26] Başbük, M., Eryılmaz, A., \& Atay, M. T., On Critical Buckling Loads of Columns under End Load Dependent on Direction. International scholarly research notices, 2014.

[27] Basbuk, M., Eryilmaz, A., Coskun, S. B., \& Atay, M. T., On Critical Buckling Loads of Euler Columns With Elastic End Restraints. Hittite Journal of Science \& Engineering, 3(1), 2016.

[28] Pinarbasi, S., Stability analysis of nonuniform rectangular beams using homotopy perturbation method. Mathematical Problems in Engineering, 2012, 2012.

[29] Mercan, K., \& Civalek, Ö., Buckling analysis of Silicon carbide nanotubes (SiCNTs) with surface effect and nonlocal elasticity using the method of HDQ. Composites Part B: Engineering, 114, 34-45, 2017.

[30] Civalek, Ö., \& Demir, C., Buckling and bending analyses of cantilever carbon nanotubes using the euler-bernoulli beam theory based on non-local continuum model. Asian Journal of Civil Engineering, 12(5), 651-661, 2011.

[31] Akgöz, B., \& Civalek, Ö., Buckling analysis of functionally graded microbeams based on the strain gradient theory. Acta Mechanica, 224(9), 2185-2201, 2013.

[32] Yayli, M. Ö., Buckling analysis of a microbeam embedded in an elastic medium with deformable boundary conditions. Micro \& Nano Letters, 11(11), 741-745, 2016.

[33] Yayli, M. Ö., Buckling analysis of a cantilever single-walled carbon nanotube embedded in an elastic medium with an attached spring. Micro \& Nano Letters, 12(4), 255-259, 2017. 
[34] Yayl1, M. Ö., Stability analysis of gradient elastic microbeams with arbitrary boundary conditions. Journal of Mechanical Science and Technology, 29(8), 3373-3380, 2015.

[25] Robinson, M. T. A., \& Adali, S., Buckling of nonuniform and axially functionally graded nonlocal Timoshenko nanobeams on Winkler-Pasternak foundation. Composite Structures, 206, 95-103, 2018.

[36] Akgöz, B., \& Civalek, Ö., Strain gradient elasticity and modified couple stress models for buckling analysis of axially loaded micro-scaled beams. International Journal of Engineering Science, 49(11), 1268-1280, 2011.

[37] Huang, Y., Yang, L. E., \& Luo, Q. Z., Free vibration of axially functionally graded Timoshenko beams with non-uniform cross-section. Composites Part B: Engineering, 45(1), 1493-1498, 2013.

[38] Huang, Y., \& Luo, Q. Z., A simple method to determine the critical buckling loads for axially inhomogeneous beams with elastic restraint. Computers \& Mathematics with Applications, 61(9), 2510-2517, 2011.

[39] Nejad, M. Z., Hadi, A., \& Rastgoo, A., Buckling analysis of arbitrary two-directional functionally graded Euler-Bernoulli nano-beams based on nonlocal elasticity theory. International Journal of Engineering Science, 103, 1-10, 2016.

[40] Pinarbasi, S., Buckling analysis of nonuniform columns with elastic end restraints. Journal of Mechanics of Materials and Structures, 7(5), 485-507, 2012.

[41] Şimşek, M., Buckling of Timoshenko beams composed of two-dimensional functionally graded material (2D-FGM) having different boundary conditions. Composite Structures, 149, 304-314, 2016.

[42] Gül, U., Aydoğdu, M., \& Edirme, E., Elastik Zemin Üzerinde Oturan Timoshenko Kirişlerinde Dalga Yayınımı, 2015.

[43] Zhang, Y., Zhang, L. W., Liew, K. M., \& Yu, J. L., Buckling analysis of graphene sheets embedded in an elastic medium based on the kp-Ritz method and non-local elasticity theory. Engineering Analysis with Boundary Elements, 70, 31-39, 2016.

[44] Yayli, M. Ö., Buckling analysis of Euler columns embedded in an elastic medium with general elastic boundary conditions. Mechanics Based Design of Structures and Machines, 46(1), 110-122, 2018.

[45] Timoshenko, S. P., \& Gere, J. M., Theory of elastic stability, 1961.

[46] Wang, C. M., \& Wang, C. Y., Exact solutions for buckling of structural members (Vol. 6). CRC press, 2004. 